\title{
The Causes of Hyperreflective Dots in Optical Coherence Tomography Excluding Diabetic Macular Edema and Retinal Venous Occlusion ${ }^{\S}$
}

\author{
Burak Turgut ${ }^{*}$ and Hakan Yildirim
}

Firat University, School of Medicine, Department of Ophthalmology, Elazı̆̆, Turkey

\begin{abstract}
Purpose: To investigate the causes of hyperreflective dots (HRDs) in spectral domain optical coherence tomography (OCT) excluding diabetic macular edema (DME) and RVO (retinal vein occlusion).

Patients and Methods: The medical records of 56 patients with HRDs documented by OCT were reviewed retrospectively. The patients with DME and RVO were excluded from the study in order to prevent misdiagnosing hard exudates or HRDs. The causes, unilaterality or bilaterality of HRD and demographic properties of the patients with HRD were evaluated.

Results: Sixty four eyes of 56 patients having HRDs were included in this study. Of the patients with HRD, 17 (30.36\%) were women and 39 (69.64\%) were men. The ages of patients were between 13 to 84 years (median 60.18 years). The causes of HRD were as follows: papilledema in 4 eyes $(6.25 \%)$, active neovascular age related macular degeneration (AMD) in 33 eyes (51.56\%), familial dominant drusen in 2 eyes $(3.13 \%)$, central serous chorioretinopathy in 19 eyes $(29.69 \%)$ and commotio retina in 2 eyes $(3.13 \%)$, choroidal folds in one eye $(1.56 \%)$, branch retinal artery occlusion in one eye $(1.56 \%)$, central retinal artery occlusion in one patient (1.56\%) and Best vitelliform macular dystrophy in one eye $(1.56 \%)$. The most common cause of HRD was AMD. The causes of HRDs in both eyes were AMD and papilledema.
\end{abstract}

Conclusion: The most common causes of HRDs excluding DME and RVO seem as active exudative AMD. The presence of HRDs in retinal diseases might affect the decisions and the results of the treatment and the prognosis of diseases.

Keywords: Hyperreflective dots, causes, optical coherence tomography.

\section{BACKGROUND}

Spectral domain optical coherence tomography (SDOCT) is a very useful non-invasive imaging method which is used in the diagnosis and follow-up of diseases involving the macula. It provides detailed information for the evaluation of drusen, intraretinal/subretinal hemorrhage or fluid and detachment of retina pigment epithelium (RPE) or retina [14]. Recently, Coscas et al. [5-7] found a new hyperreflective sign in SD-OCT previously unseen intraretinal changes and they called it as the hyperreflective dots (HRDs). They suggested that the presence of HRDs could affect the prognosis and treatment decisions, particularly in the patients with age related macular degeneration (AMD). Coscas et al. detected that HRDs scattered throughout all retinal layers, primarily around fluid accumulation in the intraretinal cystoid spaces. However, subsequently, HRDs have also been reported in retinal vein occlusion (RVO) and diabetic macular edema (DME) [7-10]. HRDs are not visible in any monochromatic and angiographic photos. Thus, we excluded the patients with DME and RVO in order to prevent misdiagnosing hard exudates or HRDs [11, 12]. In our study,

*Address correspondence to this author at the Frrat University, School of Medicine, Department of Ophthalmology, 23119, Elazığ, Turkey; Tel: +90 424 2333555; Fax: +90 424 2388096; E-mail: drburakturgut@gmail.com

${ }^{\S}$ Presented at the 14th ESASO Retina Academy, Istanbul, Turkey, November 2014. we aimed to investigate the causes of HRDs in optical coherence tomography excluding DME and RVO.

\section{PATIENTS AND METHODS}

In the study, we retrospectively reviewed the medical records of 632 patients with exlusion criteria who underwent OCT examination for various macular pathologies at our university hospital. The files were retrieved in the databases of the OCT machines. Sixty four eyes of 56 patients having HRDs were included in this study. The patients underwent complete ophthalmic examination, fundus photography, fundus autofloresence (FAF) and angiography, and OCT. OCT examinations were performed using SD-OCT and scanning laser ophthalmoscope (SLO) (OCT/SLO; OTI/OPKO Inc, Toronto, Canada). The SD-OCT device used in our study had transverse and axial resolutions of 20 and 5-6 $\mu \mathrm{m}$, respectively. The acquisition speed was set at 28000 A-scan/sec. Every B-scan was composed of 512 Ascans. In vivo OCT images with high-resolution were obtained and analyzed for the presence of singular round or oval HRDs. Additionally, the characteristics of the HRD were also evaluated in all SD-OCT scans obtained. The presence of HRD in OCT was defined as the presence of small focal hyperreflective lesions scattered mainly in outer retinal layers, but also spreading to all retinal layers, observed in at least one OCT B-scan with high signal/noise ratio $[5,6]$. HRDs were detected using only OCT while they were not visible in any red-free or chromatic photos, FAF images and angiography images. HRDs were classified on 
the basis of localization (outer, middle, inner retinal layers or disseminated in all retinal layers).

This study was designed as an institutional, retrospective trial and followed the tenets of the Declaration of Helsinki.

No approval from the institutional ethic committee board was needed due to the retrospective nature of the study. Informed consents were obtained from each patient after explanation of the nature and possible consequences of the study.

\section{Exclusion Criteria}

The patients with DME, RVO, epiretinal membrane or vitreomacular traction documented by OCT, and media opacities such as corneal opacity, lens opacity, vitreous and preretinal hemorrhage, proliferative diabetic retinopathy, arterial macroaneurysm, x-ray irradiation, carotid artery obstruction, sickle cell hemoglobinopathies, and patients with history of previous intraocular surgery, macular laser photocoagulation, and intravitreal injection were excluded from the study.
The patients had underwent complete ophthalmic examination, including best-corrected VA measurement using the Early Treatment Diabetic Retinopathy Study chart, slit-lamp biomicroscopy with a +90 diopter noncontact lens, red-free and color fundus photography, fundus autofloresence, fundus florescein angiography, and OCT.

\section{RESULTS}

Sixty four eyes of 56 patients having HRDs were included in this study. Of the patients with HRD, 17 $(30.36 \%)$ were women and $39(69.64 \%)$ were men. The ages of patients were between 13 to 84 years (median 60.18 years). The causes of HRD were as follows: papilledema in 4 eyes $(6.25 \%)$, active neovascular AMD in 33 eyes (51.56\%), familial dominant drusen (FDD) in 2 eyes $(3.13 \%)$, CSCR in 19 eyes $(29.69 \%)$ and commotio retina in 2 eyes $(3.13 \%)$, choroidal fold in one eye $(1.56 \%)$, branch retinal artery occlusion (BRAO) in one eye $(1.56 \%)$, central retinal artery occlusion (CRAO) in one patient $(1.56 \%)$ and Best's vitelliform macular dystrophy (BVMD) in one eye (1.56\%) (Table 1). The most common causes of HRD were AMD and
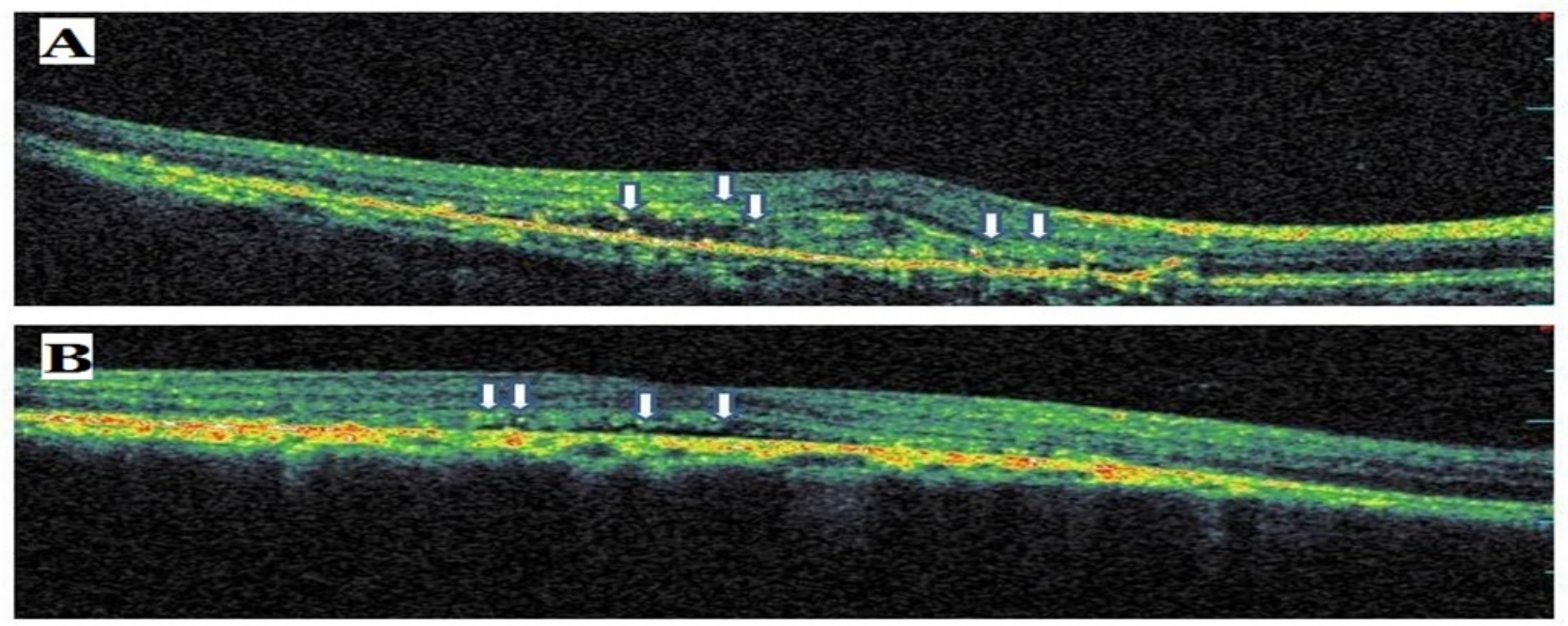

Fig. (1). Sample optical coherence tomography scans from patients with age related macular degeneration. The arrows indicate hyperreflective dots.
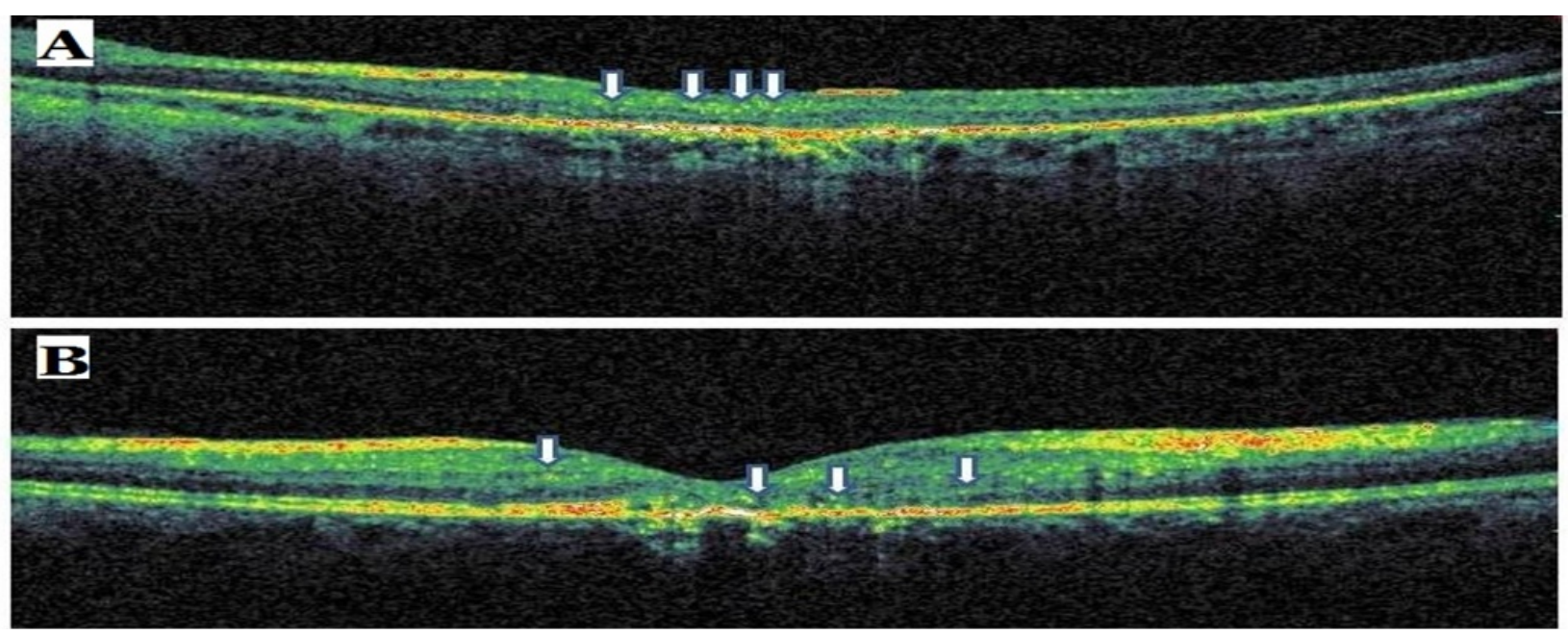

Fig. (2). Sample optical coherence tomography scans from patients with commotio retina (A) and central retinal artery occlusion (B). The arrows indicate hyperreflective dots. 

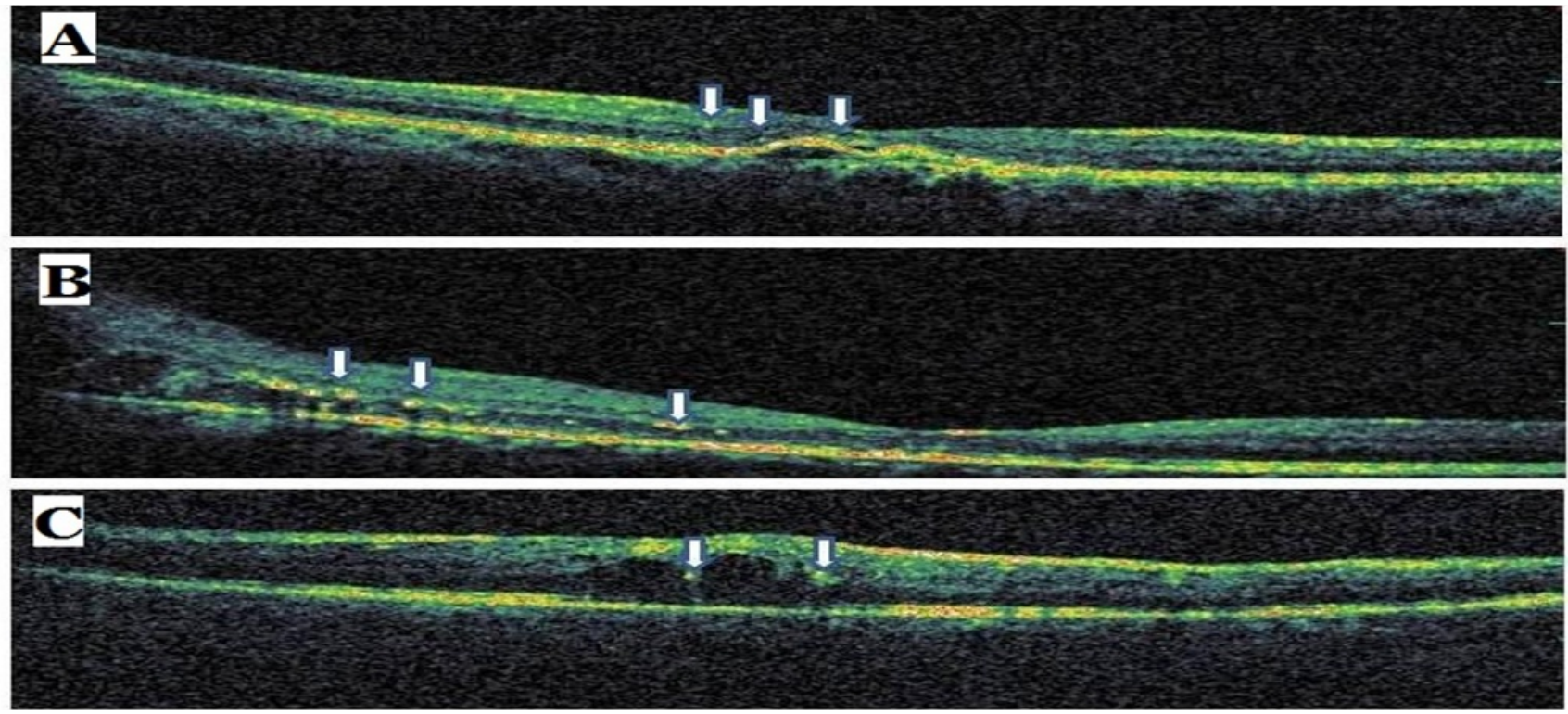

Fig. (3). Sample optical coherence tomography scans from patients with familial dominant druzen (A), papilledema (B) and branch retinal artery occlusion $(\mathbf{C})$. The arrows indicate hyperreflective dots.


Fig. (4). Sample optical coherence tomography scans from patients with central serous chorioretinopathy. The arrows indicate hyperreflective dots.

CSCR. The causes of HRDs in both eyes were AMD and papilledema. All neovascular AMD cases, HRD were at active phase and were located outer retinal layers. In the present study, all CSCR patients have seen HRD were an acute phase of the disease with serous macular detachment (SMD). In these cases, HRDs were located at outer retinal layers just over SMD. In the cases of commotio retina, HRDs were detected at middle retinal layers. HRDs were dominantly located outer retinal layers in the eyes with papilledema, BRAO, CRAO, FDD, choroidal folds and BVMD. In the case with BVMD, HRDs were arranged in outer retinal layers over SMD as seen at CSCR. The diameters of HRDs were ranging between 10 and 70 micrometers on SD-OCT images. HRDs having small diameters were usually located in the inner retinal layers while large HRDs were located in the outer retinal layers and they created the high shadowing effects. The samples of OCT scan from the eyes with HRD were given in Figs. (1-5).

\section{DISCUSSION}

The hyperreflective foci or dots are two different terms used to call abnormal hyperreflective elements detected in OCT scans. The HRDs are scattered, punctiform, small in size, mainly located in the outer retinal layers and/or around pockets of fluid accumulation and are typically not confluent [3].

HRDs are a new OCT findings demonstrated firstly by Coscas et al. [7]. It has been reported that they might seem in different retinal diseases, including AMD, DME, RVO, central serous chorioretinopathy, uveitis and macular telangiectasia [1316]. They hypothesized that HRD were detected in the majority of patients affected by exudative AMD could be inflammatory activated and swelled cells or activated microglia cells because they may spread in all retinal layers in contrast to hard exudates, that they rapidly disappear after antiVEGF or anti-inflammatory treatment [7]. 

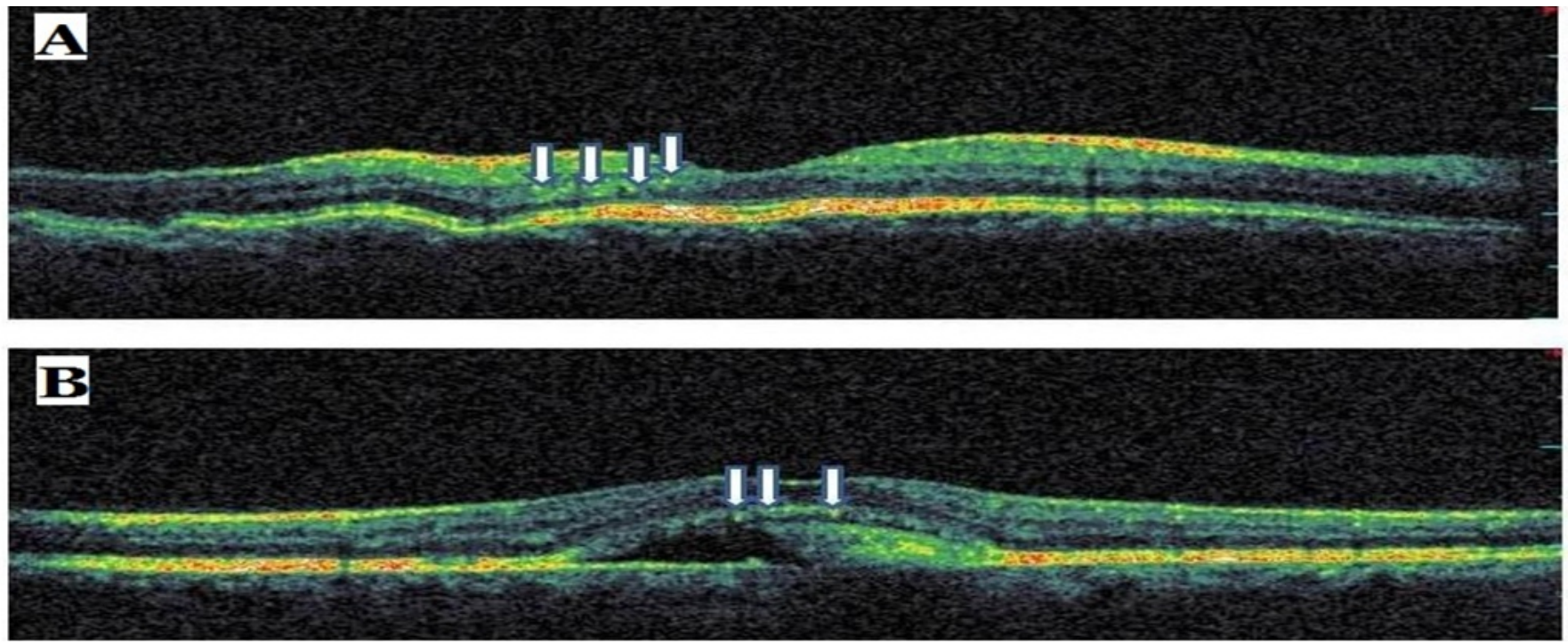

Fig. (5). Sample optical coherence tomography scans from patients with choroidal folds (A) and Best vitelliform dystrophy (B). The arrows indicate hyperreflective dots.

Although there are several theories of pathogenesis of HRD, its etiology is not clear. Some authors hypothesize that HRDs are focal accumulations of pigment or lipofuscin granules, while as others believe that they could be small intraretinal proteins or lipid exudates/deposits due to the breakdown of the bloodretinal barrier (BRB) in retinal vascular disease [7, 9, 16, 17]. Some different imaging modalities such as blue-light fundus photography and fundus autofluorescence eliminated that HRDs were not the focal accumulation of pigment or lipofuscin granules [7]. To another theory, it is considered that HRDs might be derived the degenerated photoreceptors or the macrophages fagosited them [16-19]. In a recent study, Uji et al. [19] demonstrated the presence of HRDs in the outer retinal layers and especially in the inner retinal layers in eyes with DME. They reported that HRDs in the outer retinal layers were closely associated with disrupted external limiting membrane and IS/OS line and decreased visual acuity.

Table 1. The causes of hyperreflective dots in spectral domain optical coherence tomography.

\begin{tabular}{|c|c|}
\hline HRD Cause & Eyes $(\%)$ \\
\hline \hline Papilledema & 4 eyes $(\% 6.25)$ \\
\hline Active neovascular AMD & 33 eyes $(51.56 \%)$ \\
\hline FDD & 2 eyes $(3.13 \%)$ \\
\hline CSC & 19 eyes $(29.69 \%)$ \\
\hline Commotio retina & 2 eyes $(3.13 \%)$ \\
\hline Choroidal folds in & 1 eye $(1.56 \%)$ \\
\hline BRAO & 1 eye $(1.56 \%)$ \\
\hline CRAO & 1 eye $(1.56 \%)$ \\
\hline BVMD & 1 eye $(1.56 \%)$ \\
\hline
\end{tabular}

HRDs, hyperreflectice dots; AMD, age related macular degeneration; FDD, familial dominant drusen; CSC, central serous chorioretinopathy; CRAO, central retinal artery occlusion; BRAO, branch retinal artery occlusion; BVMD, Best vitelliform macular dystrophy.

Bolz et al. [9] reported that HRDs were distributed throughout all retinal layers in DME. They demonstrated that
HRDs which located at the border of the outer nuclear layer and within the outer plexiform layer in some cases are confluent. They hypothesized that HRDs might represent subclinical features of lipoprotein extravasation that act as precursors of hard exudates, as they were not observed on clinical examination, fundus photography, or fluorescein angiography, due to their small size [9].

Ogino et al. [8] reported the presence and distribution of HRS in RVO and that HRDs were present in all retinal layers. They demonstrated that HRDs were attached to the external limiting membrane in most of the eyes with serous retinal detachment. The authors did not find any sign of hard exudates. They also suggested that the HRDs seen in affected areas might explain the leakage of blood constituents, whereas the HRS around the outer plexiform layer in the unaffected areas in RVO were associated with the absorption of water and solutes [8].

Framme et al. [20] reported the presence of HRDs in patients with both focal DME and diffuse DME. After antiVEGF treatment, the HRDs were the first features to disappear or to reduce significantly. Therefore, it has been hypothesized that HRDs represent a clinical marker of inflammatory response $[16,19]$.

In our study, we found that the most common causes of HRD were AMD and CSCR. The all eyes having HRDs were the eyes with neovascular AMD at active phase and they were located in outer retinal layers. We support to Coscas et al. [7] concerning that the HRDs might be activated inflammatory or microglia cells because it is considered that inflammation plays in the role at the pathogenesis of AMD. In the present study, all CSCR patients with HRDs were an acute phase of the disease with serous macular detachment (SMD). HRDs were located at outer retinal layers just over SMD. We observed that HRDs were at middle retinal layers in the cases of commotio retina. HRDs were dominantly located outer retinal layers in the eyes with papilledema, BRAO, CRAO, FDD, choroidal folds and BVMD. In the case of the BVMD, HRDs were arranged in outer retinal layers over SMD as seen at CSCR. 
It is known that CSCR and BVMD are non-inflammatory diseases. So, it is possible that HRDs seen in these diseases might be activated intraretinal microglia cells or macrophages phagosited outer segments of damaged photoreceptors. On the other hand, the cases with FDD, choroidal folds and commotio retina, HRDs might be due to inflammatory cells because of possible inflammatory pathogenesis of these disorders. In the cases of papilledema, BRAO and CRAO, the cause of HRDs might be the photoreceptor damage or the ischemia of the inner retinal layers.

In conclusion, the most common causes of HRDs excluding DME and RVO seem as active exudative AMD. The presence of HRDs in retinal diseases might affect the decisions and the results of the treatment and the prognosis of diseases.

\section{CONFLICT OF INTEREST}

The authors confirm that this article content has no conflict of interest.

\section{ACKNOWLEDGEMENTS}

Burak Turgut, MD, Department of Ophthalmology, Firat University Hospital: conception, design, editing, analysis and review of the manuscript.

Hakan Yildirim, MD, Assistant of Ophthalmology, Frrat University Hospital: conception, design, typing, interpretation of data and review of the manuscript.

\section{REFERENCES}

[1] Adhi M, Duker JS. Optical coherence tomography--current and future applications. Curr Opin Ophthalmol 2013; 24: 213-21.

[2] Wolf S, Wolf-Schnurrbusch U. Spectral-domain optical coherence tomography use in macular diseases: a review. Ophthalmologica 2010; 224: 333-40.

[3] Figurska M, Robaszkiewicz J, Wierzbowska J. Optical coherence tomography in imaging of macular diseases. Klin Oczna 2010; 112: $138-46$.

[4] Gabriele ML, Wollstein G, Ishikawa H, et al. Optical coherence tomography: history, current status, and laboratory work. Invest Ophthalmol Vis Sci 2011; 52: 2425-36.

[5] Coscas G, Coscas F, Vismara S, Zourdani A, Li Calzi CI. Clinical features and natural history of AMD. In: Coscas G, Coscas F, Vismara S, Zourdani A, Li Calzi CI, Eds. Optical Coherence Tomography in Age-Related Macular Degeneration. Heidelberg: Springer 2009: pp. $171-4$.
[6] Coscas G, De Benedetto U, Coscas F, et al. Hyperreflective dots: a newspectral-domain optical coherence tomography entity for follow-up and prognosis in exudative age-related macular degeneration. Ophthalmologica 2013; 229: 32-7.

[7] Coscas G, Loewenstein A, Augustin A, et al. Management of retinal vein occlusion consensus document. Ophthalmology 2011; 226: 4-28.

[8] Ogino K, Murakami T, Tsujikawa A, et al. Characteristics of optical coherence tomographic hyperreflective foci in retinal vein occlusion. Retina 2012; 32: 77-85.

[9] Bolz M, Schmidt-Erfurth U, Deak G, et al. Diabetic Retinopathy Research Group Vienna. Optical coherence tomographic hyperreflective foci: a morphological sign of lipid extravasation in diabetic macular edema. Ophthalmology 2009; 116: 914-20.

[10] Omri S, Behar-Cohen F, de Kozak Y, et al. Microglia/macrophages migrate through retinal epithelium barrier by a transcellular route in diabetic retinopathy. Am J Pathol 2011; 179: 942-53.

[11] Ota M, Nishijima K, Sakamoto A, et al. Optical coherence tomographic evaluation of foveal hard exudates in patients with diabetic maculopathy accompanying macular detachment. Ophthalmology 2010; 117: 1996-2002.

[12] Horii T, Murakami T, Nishijima K, et al. Relationship between fluorescein pooling and optical coherence tomographic reflectivity of cystoid spaces in diabetic macular edema. Ophthalmology 2012; 119: 1047-55.

[13] Fong AH, Li KK, Wong D. Choroidal evaluation using enhanced depth imaging spectral-domain optical coherence tomography in VogtKoyanagi-Harada disease. Retina 2011;31: 502-9.

[14] Kon Y, Iida T, Maruko I, Saito M. The optical coherence tomographyophthalmoscope for examination of central serous chorioretinopathy with precipitates. Retina 2008; 28: 864-9.

[15] van Velthoven ME, Verbraak FD, Garcia PM, et al. Evaluation of central serous retinopathy with en face optical coherence tomography. Br J Ophthalmol 2005; 89: 1483-8.

[16] Vujosevic S, Bini S, Midena G, et al. Hyperreflective intraretinal spots in diabetics without and with nonproliferative diabetic retinopathy: an in vivo study using spectral domain OCT. J Diabetes Res 2013; 2013 : 491835.

[17] Gaudric A, Ducos de Lahitte G, Cohen SY, Massin P, Haouchine B. Optical coherence tomography in group 2A idiopathic juxtafoveolar retinal telangiectasis. Arch Ophthalmol 2006; 124: 1410-9.

[18] Baumüller S, Charbel Issa P, Scholl HP, Schmitz-Valckenberg S, Holz FG. Outer retinal hyperreflective spots on spectral-domain optical coherence tomography in macular telangiectasia type 2 . Ophthalmology 2010; 117: 2162-8.

[19] Uji A, Murakami T, Nishijima K, et al. Association between hyperreflective foci in the outer retina, status of photoreceptor layer, and visual acuity in diabetic macular edema. Am J Ophthalmol 2012; 153: 710-7.

[20] Framme C, Schweizer P, Imesch M, Wolf S, Wolf-Schnurrbusch U. Behavior of SD-OCT-detected hyperreflective foci in the retina ofantiVEGF-treated patients with diabetic macular edema. Invest Ophthalmol Vis Sci 2012; 53: 5814-8. 